\title{
Value of a Lower-Limb Immobilization Device for Optimization of SPECT/CT Image Fusion
}

\author{
Joana do Mar F. Machado ${ }^{1-3}$, Marina S. Monteiro², Victor Fernandes Vieira², Jean-Aybert Collinot ${ }^{2}$, John O. Prior ${ }^{2}$, \\ Lina Vieira ${ }^{3}$, and José A. Pires-Jorge ${ }^{1}$ \\ ${ }^{1}$ Haute École de Santé Vaud-Filière TRM, University of Applied Sciences and Arts Western Switzerland, Lausanne, Switzerland; \\ ${ }^{2}$ Nuclear Medicine Department, Lausanne University Hospital, Lausanne, Switzerland; and ${ }^{3}$ Área Cientifica de Medicina Nuclear, \\ Escola Superior de Tecnologia da Saúde de Lisboa, Instituto Politécnico de Lisboa, Lisbon, Portugal
}

\begin{abstract}
The foot and the ankle are small structures commonly affected by disorders, and their complex anatomy represents a significant diagnostic challenge. By providing information on anatomic and bone structure that cannot be obtained from functional imaging, SPECT/CT image fusion can be particularly useful in increasing diagnostic certainty about bone pathology. However, because of the lengthy duration of a SPECT acquisition, a patient's involuntary movements may lead to misalignment between SPECT and CT images. Patient motion can be reduced using a dedicated patient support. We designed an ankle- and foot-immobilizing device and measured its efficacy at improving image fusion. Methods: We enrolled 20 patients who underwent SPECT/CT of the ankle and foot with and without a foot support. The misalignment between SPECT and CT images was computed by manually measuring 14 fiducial markers chosen among anatomic landmarks also visible on bone scintigraphy. ANOVA was performed for statistical analysis. Results: The absolute average difference without and with support was $5.1 \pm 5.2 \mathrm{~mm}$ (mean $\pm \mathrm{SD}$ ) and $3.1 \pm 2.7 \mathrm{~mm}$, respectively, which is significant $(P<0.001)$. Conclusion: The introduction of the foot support significantly decreased misalignment between SPECT and CT images, which may have a positive clinical influence in the precise localization of foot and ankle pathology.
\end{abstract}

Key Words: SPECT/CT; misalignment; lower limb; patient motion

J Nucl Med Technol 2015; 43:98-102

DOI: 10.2967/jnmt.114.145771

\section{$\mathbf{S}$} functional and anatomic information (1-5) in the identification and characterization of different disorders (2), including endocrine and neuroendocrine diseases, infection and inflammation (2,4,6-8), and benign and malignant bone diseases $(2,4)$. SPECT/CT is currently a main, growing focus of interest in the assessment of musculoskeletal disorders (5).

\footnotetext{
Received Jul. 18, 2014; revision accepted Jan. 27, 2015.

For correspondence or reprints contact: José A. Pires-Jorge, Haute École de Santé Vaud, Avenue de Beaumont 21, CH-1011 Lausanne, Switzerland. E-mail: jose.jorge@hesav.ch

Published online Apr. 9, 2015.

COPYRIGHT (C 2015 by the Society of Nuclear Medicine and Molecular Imaging, Inc.
}

The high sensitivity provided by SPECT combined with the increased specificity provided by CT $(7,9)$ can increase accuracy and confidence in areas of special diagnostic difficulty, such as the foot and the ankle $(7,9,10)$. Indeed, in clinical examination, it can be challenging to find the origin of the pain (10) even for the most experienced of clinicians (4), mainly because of the variety of etiologies producing similar patient complaints and clinical abnormalities (8). The foot and the ankle comprise a complex anatomy of small structures $(1,9,10)$, including bones, ligaments, and tendons (11), which can be subject to inflammatory and degenerative diseases producing severe disability (12).

To make specific diagnoses and deliver appropriate treatments, small or focal pathologic changes must be well localized (7). Currently, MR imaging is the most widely used technique in evaluating chronic foot and ankle pain, although SPECT/CT can play an important role in assessing the origin of pain $(4,10)$ and early stages of disease $(13)$. On the other hand, in early degenerative changes in the varus and valgus misaligned hind foot, SPECT/CT is useful before conventional scintigraphy and CT scanning (14).

Aligning the datasets of 2 different modalities is not a simple task, because of differences in imaging resolution, patient alignment (15), and the information obtained from each technique (16).

Some authors have shown that misaligned images resulting from patient movement $(6,17)$ occur in most studies and that even a 1-pixel misalignment can be visible on corrected SPECT images (18). On the basis of CT information about tissue absorption, SPECT images can be corrected for tissue and bone attenuation. The pixel values must be appropriately scaled to match attenuation coefficients, and the SPECT images must be precisely aligned. CT-based attenuation correction is sensitive to spatial misalignment between CT and SPECT and can result in artifacts in the attenuation-corrected SPECT scan (19). To avoid these issues, it is important to optimize patient preparation, which includes appropriate and comfortable support, as well as to select established and validated protocols $(20,21)$.

The aim of this study was to manufacture and implement a dedicated foot immobilization support during SPECT/CT acquisitions of the foot and ankle to decrease the likelihood 


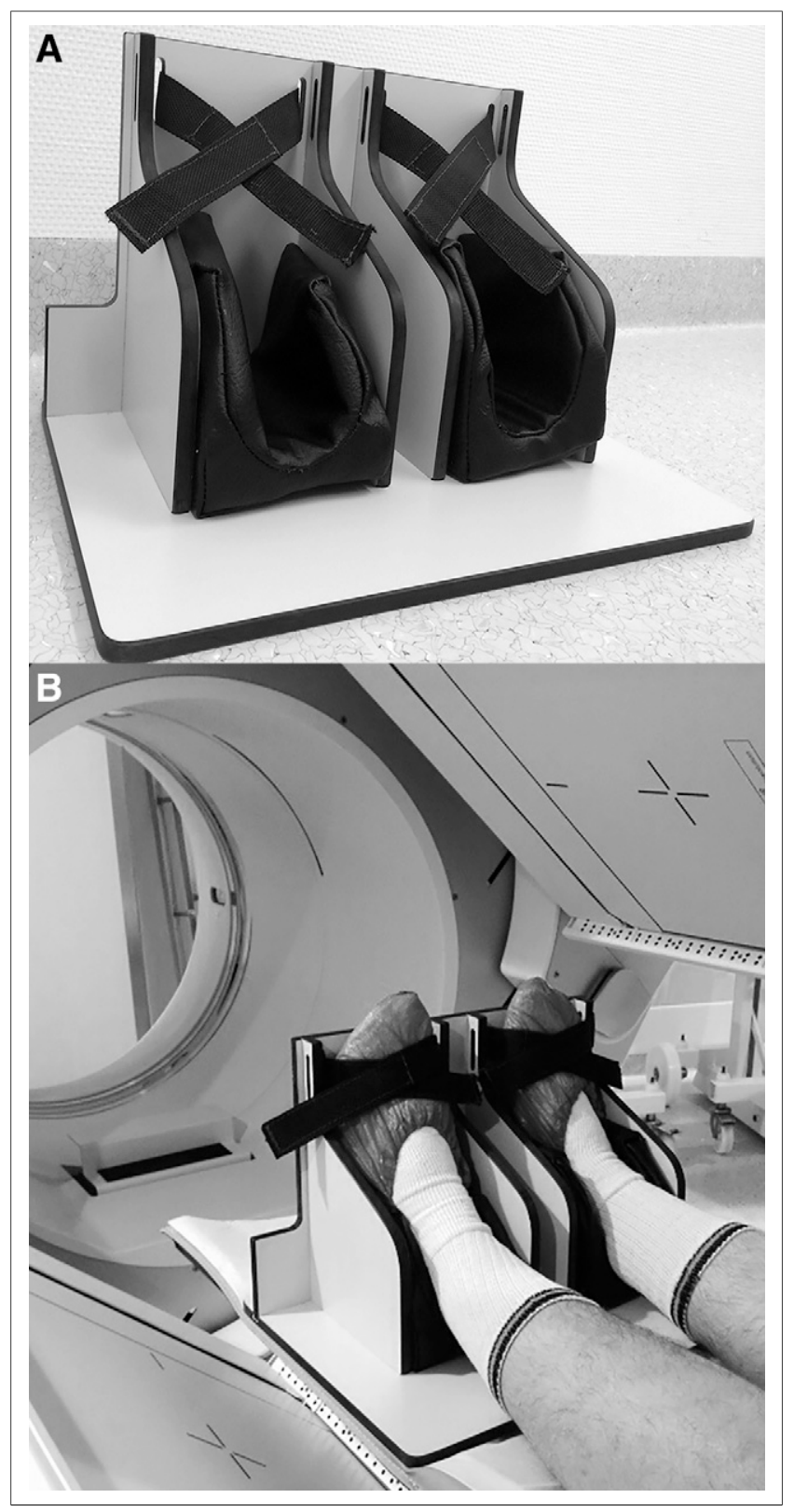

FIGURE 1. (A) Dedicated support for ankle and foot for SPECT/CT acquisitions. (B) Foot is well fixed and retains aligned position thanks to comfortable cushions.

of movement between the two studies, thus contributing to improved alignment.

\section{MATERIALS AND METHODS}

\section{Patient Population}

We evaluated 20 patients (mean age, $50 \pm 15$ y; range, $18-73$ y; 16 men and 4 women) who were referred for investigation of persistent pain in the foot or ankle after traumatic injury. Between January and May 2014, they underwent distal lower-limb SPECT/ $\mathrm{CT}$ imaging at the end of a standard 3-phase bone scintigraphy study. According to the Swiss legislation (article 2 of the Federal Act on Research Involving Human Beings), the ethics commission ruled that the present study on imaging quality did not require approval by a Swiss ethics committee on research involving humans and waived the requirement to obtain written informed consent.

The patients were divided into 2 groups: group G1 included 10 patients on whom SPECT/CT was performed without the foot support, and group G2 included 10 patients on whom SPECT/CT was performed with the foot support.

\section{Foot Support}

The support consisted of a 40-cm (length) $\times 22-\mathrm{cm}$ (width) $\times$ $15-\mathrm{cm}$ (height) compact panel composed of synthetic materials (0.8- and 1-cm thickness, 200-300 HU) built for this study to achieve firm but comfortable immobilization of the foot and ankle during the examination (Fig. 1). The inner parts were padded with soft material (2- to 3-cm thickness, $-950 \mathrm{HU}$ ) to reduce patient discomfort. The support did not significantly attenuate the $\gamma$ radiation at 140-keV energies $(\sim 13 \%-16 \%)$ (22). More important, the support was entirely in the field of view of the CT scanner so as to not introduce truncation artifacts during attenuation correction (17).

Each patient was asked to avoid moving during the procedure. The support was manufactured to resemble the ankle and foot MR imaging array coil used when needed by our radiology department to ease SPECT/MR imaging fusion. We confirmed that both feet of the patient were always securely fixed with the built-in strap to increase accuracy and to make patients feel comfortable during image acquisition.

\section{Image Acquisition}

All patients underwent classic ${ }^{99 \mathrm{~m}} \mathrm{Tc}-3,3$-diphosphono-1,2- propanedicarboxylic acid bone scintigraphy after intravenous injection of a mean ( \pm SD) activity of $840 \pm 100 \mathrm{MBq}$ (range, 590-950 $\mathrm{MBq}$ ), followed by SPECT/CT imaging of both foot and ankle at $5.4 \pm 0.9 \mathrm{~h}$ (range, 3.8-7.8 h) after tracer injection.

SPECT/CT (Discovery NM/CT 670; GE Healthcare) images were acquired using a high-resolution low-energy collimator with auto contour rotation mode ( $15 \mathrm{~s} /$ frame; 60 views over $360^{\circ}$; stepand-shoot mode, matrix size, $128 \times 128$; zoom factor, 1.00$)$ and a $15 \%$ energy window centered on $140 \mathrm{keV}$. Helical CT acquisitions used a voltage of $120 \mathrm{kV}$, a current of $90 \mathrm{~mA}$, a slice thickness of $0.625 \mathrm{~mm}$, and a 512 matrix for reconstruction.

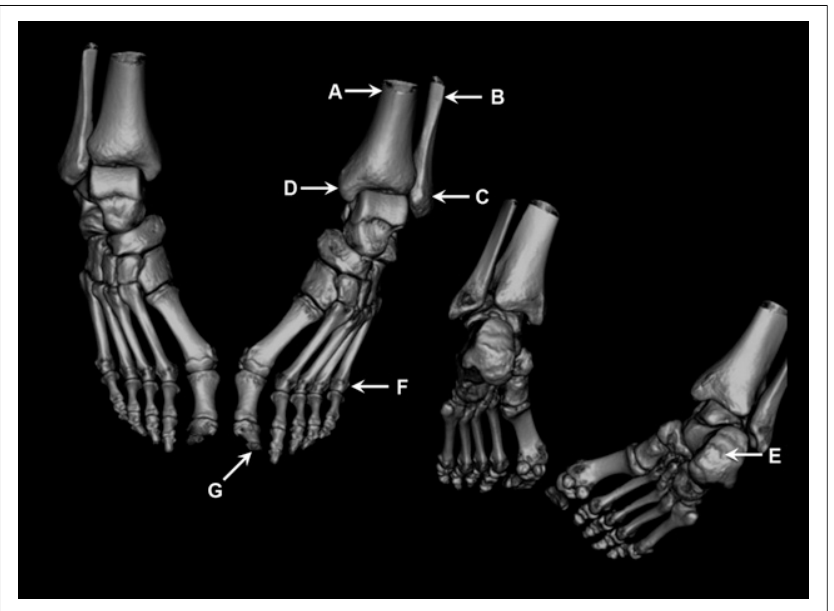

FIGURE 2. The 7 anatomic landmarks used to quantify misalignment between CT and SPECT images. $A=$ tibia; $B=$ fibula; $\mathrm{C}=$ medial malleolus; $\mathrm{D}=$ lateral malleolus; $\mathrm{E}=$ insertion of the Achilles tendon; $F=$ tuberosity of the fifth metatarsal; $\mathrm{G}=$ distal phalanx of great toe. 


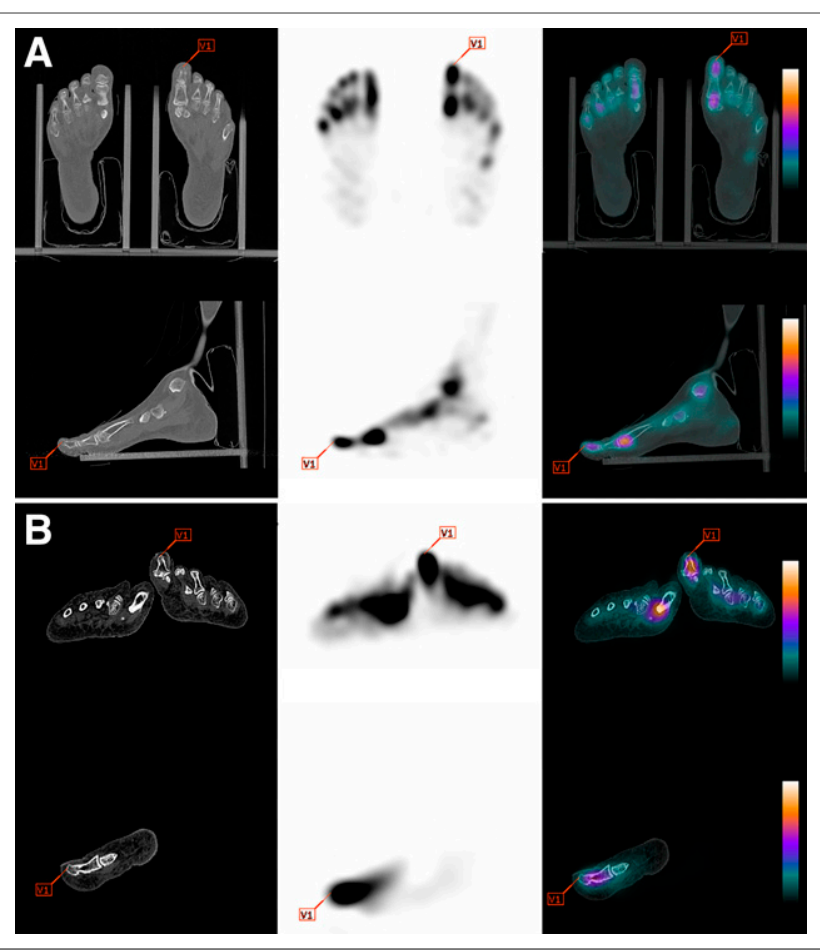

FIGURE 3. Example of anatomic landmark of left distal phalanx of great toe in patients with (A) and without (B) support. From left to right are shown CT, SPECT, and SPECT/CT fusion images.

For SPECT image reconstruction, we applied ordered-subset expectation maximization with 2 iterations and 10 subsets, using a point-spread-function recovery "Evolution for Bone" algorithm and $\mathrm{CT}$ for attenuation correction on a Xeleris 3.0 workstation (GE Healthcare).

\section{Image Analysis}

SPECT and CT image datasets were processed using the integrated registration tool on an Advantage workstation (GE Healthcare). The misalignment between SPECT and CT acquisitions was quantified by manually placing 14 fiducial markers chosen among anatomic landmarks on each SPECT and CT image (tibia, fibula, medial malleolus, lateral malleolus, insertion of the Achilles tendon, tuberosity of the fifth metatarsal, and distal phalanx of the great toe of the right and left foot and ankle) (Fig. 2). For optimal visualization of the different landmarks, we allowed varying of the color look-up table of the image. The fiducial markers were placed by a nuclear technologist and subsequently checked by 2 nuclear physicians.

\section{Statistical Analysis}

The results were analyzed using Stata 13.1 (StataCorp). Mean and SD are presented unless specified otherwise. Box plots were generated to display the absolute difference between patient groups. Differences between the 2 datasets according to the presence or absence of support and the fiducial marker number $(1-14,7$ for the right foot and 7 for the left) were assessed using ANOVA. A $P$ value of 0.05 was considered the threshold for significance.

\section{RESULTS}

In total, 280 anatomic landmarks were analyzed in 20 patients (as illustrated in Fig. 3 for the great toe landmark on SPECT and CT images for a patient with and without the support). The absolute difference for patients without and with support was $5.1 \pm 5.2 \mathrm{~mm}$ vs. $3.1 \pm 2.7 \mathrm{~mm}$, respectively. Thus, the alignment between SPECT and CT images was better with support, as shown in Figure 4. When considering a misalignment threshold of $5 \mathrm{~mm}$ as a reference for appropriate clinical evaluation, most individuals using the support were below this empiric value (dotted line), as opposed to patients without the support.

Results of the ANOVA showed that misalignments were significantly smaller in the group using the support than in the group without support $(P<0.0001)$. Moreover, the misalignments were not dependent on the fiducial marker $(P=0.54)$ (Fig. 5A).

\section{DISCUSSION}

To our knowledge, this was the first study that quantitatively evaluated the value of a lower-limb immobilization device during SPECT/CT acquisitions. Our results showed significantly less misalignment of SPECT/CT images in patients with the dedicated foot support than in those without, presumably because of the more stable and comfortable patient position using the support. However, a certain degree of misalignment is still present because of uncontrollable movements, and the support may need to be adjusted for each patient (e.g., the location of the distal extremities of the toes depends on the foot size).

Beyer et al. assessed the effectiveness of different supports to reduce misregistration in the head and the neck during

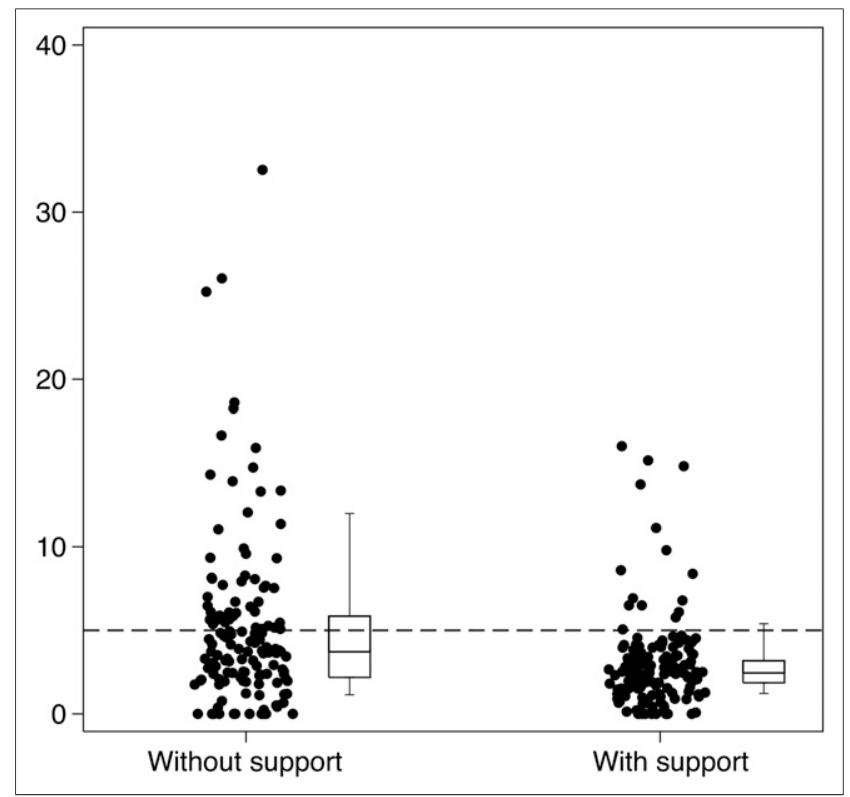

FIGURE 4. Box plot showing distribution of absolute difference |SPECT-CT| (mm) in groups of patients without and with support (box represents median, and quartiles and whiskers show 10th and 90th percentiles). Dashed line is drawn at $5 \mathrm{~mm}$ (representing the size of a SPECT voxel), above which $32 \%$ $(45 / 140)$ of measurements were larger without support and only $12 \%(17 / 140)$ with support $(P<0.001)$. 


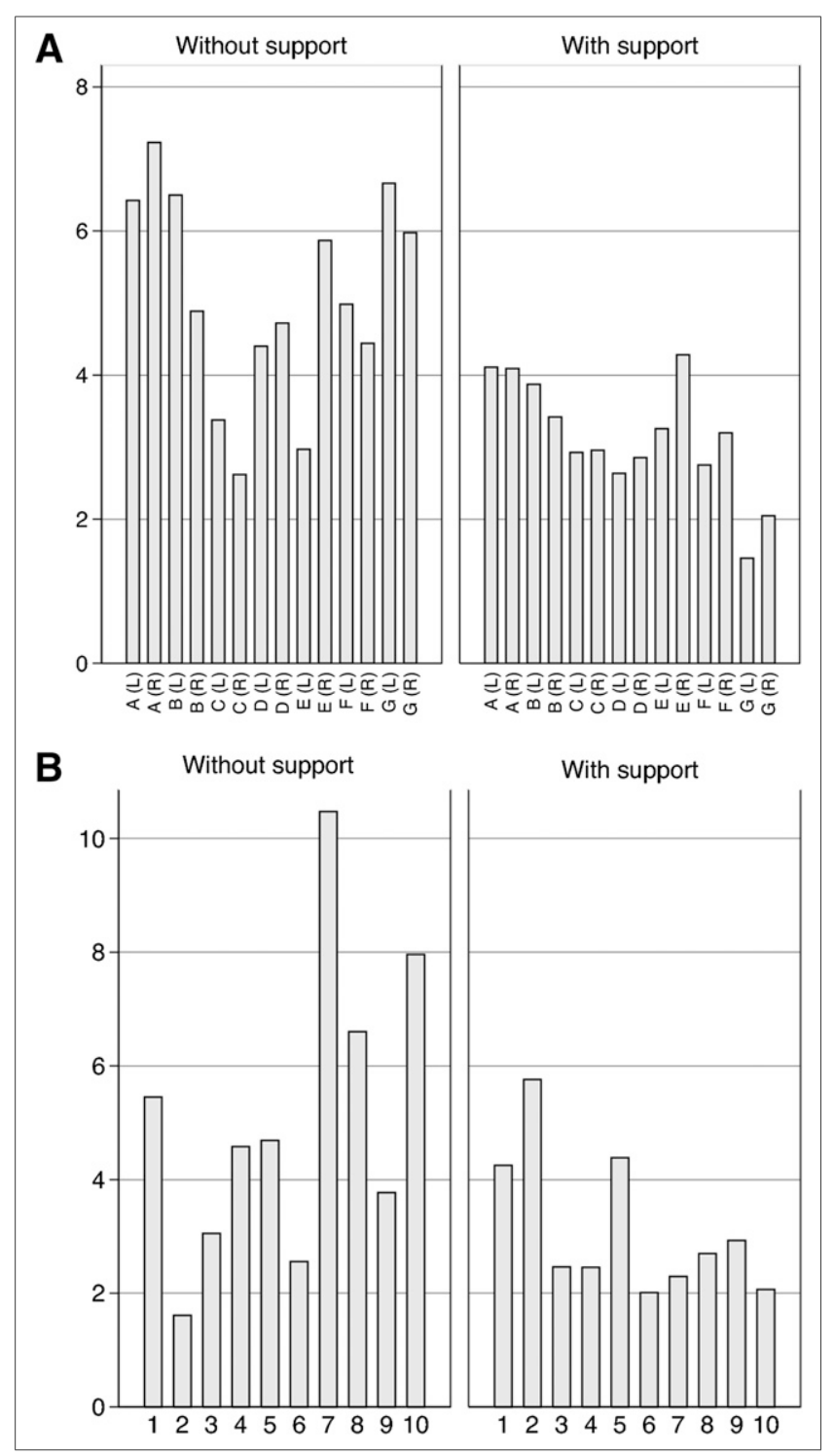

FIGURE 5. (A) Mean absolute difference $|\mathrm{SPECT}-\mathrm{CT}|(\mathrm{mm})$ according to landmarks A-G (Fig. 2) for left and right foot without and with support. (B) Same mean absolute difference |SPECT-CT| (mm) for patients 1-10 without and with support.

whole-body PET/CT studies using anatomic landmarks (21). They observed that the likelihood of motion can be reduced and accuracy improved during coregistration (the misalignment was reduced to a minimum of $1.4 \mathrm{~mm}$ for a head holder fitted with a vacuum-lock bag). However, our study showed larger misalignments even when the support was applied, as can be explained by the poorer spatial resolution and greater partial-volume effect of SPECT. Moreover, head and the neck structures may be more stable than such lower-extremity structures as those addressed in this article.

An important limitation of manual alignment is the focally increased pathologic uptake observed in certain patients, which increases uncertainty in assigning anatomic landmarks near these structures. The simultaneous analysis of CT images increases accuracy with this approach, emphasizing the previously mentioned limitation. Structures such as malleoli or the insertion of the Achilles tendon were particularly difficult to identify, but the task seemed easier when the support was used. In these conditions, the support seems to improve the clinical performance of the test, allowing for better characterization of small focal abnormalities. The spatial resolution of SPECT was worse than that of CT, leading to difficulties in precisely identifying anatomic landmarks and possibly masking smaller differences.

Gayed et al. reported better diagnostic quality for SPECT/ CT than for SPECT (8). Therefore, there is a need to minimize misregistration between SPECT and CT using a support such as ours. For instance, in Figure 4 the horizontal dashed line at $5 \mathrm{~mm}$ represents the size of a SPECT voxel, or about half the spatial full-width-at-half-maximum SPECT resolution. For the patients without support, $32 \%(45 / 140)$ of the values are above this line, whereas only $12 \%$ (17/140) are above this line for patients with support-a significant difference $(P<0.001)$.

One limitation of the design of our current support was a slight increase in the distance between the foot/ankle and the detector due to the intrinsic geometry of the support, which was manufactured to look like a foot/ankle MR array coil to allow easy SPECT/MR fusion. The slight increase in distance could lead to a slight decrease in spatial resolution. Another limitation of our study was the relatively small number of patients in each group (10) and the high number of anatomic landmarks in each patient (14), making for a large number of measurements in each group (140). Our future research will include comparing comfort with and without support in the same patient, as well as simultaneously evaluating image quality.

\section{CONCLUSION}

Our study showed that a foot immobilization device can significantly reduce misalignment between SPECT and CT images during hybrid SPECT/CT. Use of such a support may improve localization and clinical diagnosis in areas of complex foot and ankle anatomy.

\section{DISCLOSURE}

No potential conflict of interest relevant to this article was reported.

\section{ACKNOWLEDGMENT}

We are indebted to Michel Gillieron from our institution's wood workshop for manufacturing the support.

\section{REFERENCES}

1. van Dijk M, Lavalaye J. Nuclear imaging in orthopaedic decision making with focus on ankle and foot. Tijdschr Nucl Geneesk. 2012;34:968-976.

2. Mariani G, Bruselli L, Kuwert T, et al. A review on the clinical uses of SPECT/ CT. Eur J Nucl Med Mol Imaging. 2010;37:1959-1985.

3. Jacene HA, Goetze S, Patel H, Wahl RL, Ziessman HA. Advantages of hybrid SPECT/CT vs SPECT alone. Open Med Imaging J. 2008;2:67-69. 
4. Singh VK, Javed S, Parthipun A, Sott AH. The diagnostic value of single photonemission computed tomography bone scans combined with CT (SPECT-CT) in diseases of the foot and ankle. Foot Ankle Surg. 2013;19:80-83.

5. Pagenstert GI, Barg A, Leumann AG, et al. SPECT-CT imaging in degenerative joint disease of the foot and ankle. J Bone Joint Surg Br. 2009;91:1191-1196.

6. Filippi L, Uccioli L, Giurato L, Schillaci O. Diabetic foot infection: usefulness of SPECT/CT for ${ }^{99 m}$ Tc-HMPAO-labeled leukocyte imaging. J Nucl Med. 2009;50:1042-1046.

7. Scharf S. SPECT/CT imaging in general orthopedic practice. Semin Nucl Med. 2009;39:293-307.

8. Gayed I, Wan D, Joseph U, Awad J, John S. Impact of bone SPECT-CT imaging on evaluation of lower extremities' pathology [abstract]. J Nucl Med. 2011;52(suppl 1):456.

9. Huellner MW, Strobel K. Clinical applications of SPECT/CT in imaging the extremities. Eur J Nucl Med Mol Imaging. 2014;41(suppl 1):S50-S58.

10. Mohan HK, Gnanasegaran G, Vijayanathan S, Fogelman I. SPECT/CT in imaging foot and ankle pathology: the demise of other coregistration techniques. Semin Nucl Med. 2010;40:41-51.

11. Navas A. Role of computed tomography in chronic ankle and foot pain. Tijdschr Nucl Geneesk. 2012;34:961-967.

12. Robinson AH, Bird N, Screaton N, Wraight EP, Meggitt BF. Coregistration imaging of the foot: a new localisation technique. J Bone Joint Surg Br. 1998;80:777-780.

13. Knupp M, Pagenstert GI, Barg A, Bolliger L, Easley ME, Hintermann B. SPECT-CT compared with conventional imaging modalities for the assessment of the varus and valgus malaligned hindfoot. J Orthop Res. 2009;27:1461-1466.

14. Saha S, Burke C, Desai A, Vijayanathan S, Gnanasegaran G. SPECT-CT: applications in musculoskeletal radiology. Br J Radiol. 2013;86:20120519.
15. Psiuk-Maksymowicz K, Borys D, Gorczewski K, Steinhof K, d'Amico A. CT/ SPECT image fusion in patients treated with iodine-131. J Med Informatics \& Technologies. 2004;8:7-13.

16. Majumder DD, Ray D. Approaches of multimodal medical images registration and fusion: efficacy on diagnostic and therapeutic planning. IETE $J$ Res. 2011;57:498-514.

17. Płachcińska A, Siennicki J, Kovacevic-Kuśmierek K, Bieńkiewicz M, Kuśmierek J. Effect of attenuation correction on normal ${ }^{99 \mathrm{~m}} \mathrm{Tc}-\mathrm{MIBI}$ myocardial perfusion scintigrams acquired with a hybrid SPECT/CT camera. Nuclear Med Rev. 2008;11:59-66.

18. Fricke H, Fricke E, Weise R, Kammeier A, Lindner O, Burchert W. A method to remove artifacts in attenuation-corrected myocardial perfusion SPECT introduced by misalignment between emission scan and CT-derived attenuation maps. J Nucl Med. 2004;45:1619-1625.

19. Seo Y, Mari C, Hasegawa BH. Technological development and advances in single-photon emission computed tomography/computed tomography. Semin Nucl Med. 2008;38:177-198.

20. Gnanasegaran G, Cook G, Adamson K, Fogelman I. Patterns, variants, artifacts, and pitfalls in conventional radionuclide bone imaging and SPECT/CT. Semin Nucl Med. 2009;39:380-395.

21. Beyer T, Tellmann L, Nickel I, Pietrzyk U. On the use of positioning aids to reduce misregistration in the head and neck in whole-body PET/CT studies. J Nucl Med. 2005;46:596-602.

22. Brown S, Bailey DL, Willowson K, Baldock C. Investigation of the relationship between linear attenuation coefficients and CT Hounsfield units using radionuclides for SPECT. Appl Radiat Isot. 2008;66:1206-1212. 